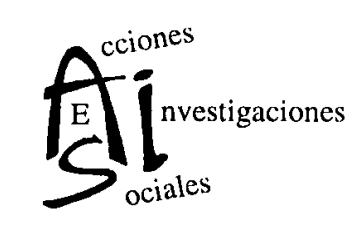

\title{
El análisis de cuotas de mercado constantes en la literatura económica internacional
}

\author{
Ana Belén Gracia Andía \\ DEPARTAMENTO DE ESTRUCUTURA \\ E HISTORIA ECONÓMICA Y PÚBLICA
}

FACULTAD DE CIENCIAS ECONÓMICAS Y EMPRESARIALES

UNIVERSIDAD DE ZARAGOZA

El artículo presentado es una revisión de la literatura económica internacional sobre el análisis de cuotas de mercado constantes, más conocido como Constant Market Shares Analysis (CMS). Este método analiza el crecimiento de los flujos comerciales de exportación de un país en un determinado período de tiempo y descompone dicha variación en dos efectos básicos, el primero asociado a fuerzas de demanda y el segundo a variables de oferta1. En el último apartado el trabajo se aproxima a los antecedentes españoles que han aplicado la técnica a las exportaciones nacionales.

Palabras clave: análisis de cuotas de mercado constantes, sector exterior, exportaciones. 


\section{El análisis de cuotas de mercado constantes en la literatura Ana Belén Gracia Andía económica internacional}

\section{Antecedentes extranjeros}

La metodología de cuotas de mercado constantes ha sido utilizada a lo largo de los últimos decenios del siglo XX por numerosos autores en estudios para el análisis del sector exterior de diferentes países y áreas económicas, y de distintos períodos de tiempo de mayor o menor amplitud, que han abarcado desde finales del XIX hasta la actualidad.

El primer autor que utilizó el método en el contexto del sector exterior para analizar el crecimiento de las relaciones comerciales de los países industrializados producido durante la primera mitad del siglo xx fue Tyszynski en $1951^{2}$. Los países a los que se refirió su análisis fueron once: Reino Unido, Estados Unidos,

${ }^{1}$ La expresión simplificada del método es la siguiente:

$$
\Delta x=x^{1}-x^{0}=s^{0} \cdot \Delta X+X^{1} \cdot \Delta s
$$

donde $x$ son las exportaciones del país objeto de estudio, $X$ las exportaciones mundiales y s la cuota de exportación de dicho país.La versión ampliada de este modelo, el más utilizado en la literatura económica, es la presentada a continuación:

$$
\Delta x=s^{0} \cdot \Delta X+\left[\sum_{i} s_{i}{ }^{0} \cdot \Delta X_{i}-s^{0} \cdot \Delta X\right]+\left[\sum_{i} \sum_{j} s i j^{0} \cdot \Delta X_{i j-} \sum_{i} s_{i}{ }^{0} \cdot \Delta X\right]+\sum_{i} \sum_{j} X_{i j}{ }^{1} \cdot \Delta s_{i j}
$$

donde el subíndice $i$ representa al producto i-ésimo de la cesta exportadora y el subíndice $j$ el destino j-ésimo de las ventas.

2 No obstante, ya en 1943, Creamer utilizó esta metodología de cuotas constantes aplicada a un trabajo más amplio sobre localización industrial, Shifts of Manufacturing Industries, y no sobre comercio exterior. 
Francia, Alemania, Bélgica, Italia, Suecia, Suiza, Canadá, India y Japón. Los grupos de productos manufacturados que incluyó en la base de datos fueron dieciseis, y los años elegidos para calcular los efectos cinco, que cubrían el período 1899-1950: 1899, 1913, 1929, 1937 y 1950.En su estudio se ocupó de analizar tanto los cambios de las exportaciones como de las importaciones, igual que han hecho otros autores, pero el trabajo se limita a presentar los resultados derivados de la evolución de las primeras por ser el objetivo más común de la mayoría de las aplicaciones.

Para él las razones de la variación del volumen de las ventas al exterior de un país se pueden reducir a dos. Por un lado, puede alterarse la importancia relativa de alguno de los grupos de productos en el total del comercio mundial, variación que representa un cambio en la estructura del comercio. Por otro lado, puede cambiar la cuota de mercado de las exportaciones de ese país en el total de las exportaciones mundiales, lo que supondría un cambio en la competitividad de dicho país.

El procedimiento de cálculo utilizado para obtener en qué medida es un efecto $u$ otro el responsable de la variación es algo diferente al considerado por el modelo más tradicional del análisis de cuotas de mercado constantes, pero el supuesto base es exactamente el mismo. Para conseguir ambos efectos Tyszynski calcula lo que él llamó la cuota «hipotética» $\left(\mathrm{s}^{\mathrm{H}}\right)$ de cada país en el comercio mundial, bajo el supuesto de que durante el período elegido todos los países mantengan su posición competitiva inicial en cada grupo.

La diferencia entre esa cuota hipotética en el año 1 (último año del período) y su cuota real en el año 0 (primer año del período) es la cuota ganada o perdida debido al cambio de estructura. Y la diferencia entre la cuota real en el año 1 y la cuota hipotética es la variación asignada al efecto competitividad.

Siendo ${ }^{3}$ :

$$
s^{0}=\frac{x^{0}}{X^{0}}=\frac{\sum_{i} s_{i}{ }^{0} \cdot X_{i}{ }^{0}}{\sum_{i} X_{i}{ }^{0}}
$$

${ }^{3}$ Se mantiene la notación utilizada en las identidades de la nota 1. 


$$
\begin{gathered}
s^{1}=\frac{X^{1}}{X^{1}}=\frac{\sum_{i} s_{i}{ }^{1} \cdot X_{i}{ }^{1}}{\sum_{i} X_{i}{ }^{1}} \\
s^{H}=\frac{X^{H}}{X^{H}}=\frac{\sum_{i} s_{i}{ }^{0} \cdot X_{i}{ }^{1}}{\sum_{i} X_{i}^{1}}
\end{gathered}
$$

Entonces:

$$
\text { Efecto estructura }=s^{H}-s^{0}=\frac{\sum_{i} s_{i}{ }^{0} \cdot X_{i}{ }^{1}}{\sum_{i} X_{i}^{1}}-\frac{\sum_{i} s_{i}{ }^{0} \cdot X_{i}{ }^{0}}{\sum_{i} X_{i}^{0}}
$$

El procedimiento y las conclusiones de Tyszynski son similares a los obtenidos por Svennilson (1954).Este autor tomó datos de exportaciones e importaciones de Estados Unidos y siete países europeos: Reino Unido, Alemania, Francia, Italia, Bélgica-Luxemburgo, Suiza y Suecia, para los años 1913, 1928, 1938 y 1950. El sistema de clasificación de los productos no fue el CUCl, sino un sistema basado en una división utilizada por los británicos antes de 1954 y compuesta por nueve grupos. En estudios posteriores se utilizó más esta nomenclatura que la empleada por Tyszynski.

Tras estos trabajos pioneros, tiene interés el artículo elaborado por Baldwin (1968). En él analizó el crecimiento de las relaciones comerciales entre los países del mundo más importantes, añadió a los datos utilizados por Svennilson las exportaciones de Canadá y Japón y amplió el período de estudio, que abarcaba desde 1900 hasta 1954. Para Baldwin el procedimiento que utilizaron Tyszynski y Svennilson para calcular los dos efectos, el de estructura y el de competitividad, atribuye al cálculo de ambos diferentes pesos, ya que al primero le asigna los del año 0 y al segundo los del 1, pudiendo esto sesgar los resultados ${ }^{4}$. Esta limitación está presente en otros artículos posteriores, que incluso proceden a calcular los efectos invirtiendo los pesos o asignándoles a ambos el mismo. Baldwin recogió en su

${ }^{4}$ Baldwin adelantó en este artículo una de las limitaciones que presentaba el modelo, y que ningún trabajo aplicado había tratado con anterioridad. 
artículo una sugerencia de Lichtenberg ${ }^{5}$ en la que afirmaba que si se atribuyera a ambos componentes los pesos del año 0 , se obtendría un efecto interactivo como elemento residual, que al sumarlo al elemento competitivo daría como resultado el efecto competitividad calculado por Tyszynski.

Este artículo de Baldwin es además muy interesante porque presenta una relación de trabajos sobre comercio exterior que han analizado la estructura del patrón comercial de cada país, atendiendo especialmente a los diferentes grupos de productos que lo componen y a la participación de las ventas de cada uno de ellos en el total mundial. La mayoría de los autores citados aplicó el análisis de cuotas constantes en sus trabajos, aunque fuese de una manera indirecta o complementaria. Hilgerdt (1945) es la primera referencia de Baldwin. Este autor ya en 1942 realizó un primer trabajo cuya elaboración contó con muchos obstáculos, pero el más conocido fue el publicado en 1945, en el que midió la evolución de los datos de comercio mundial por países desde 1871 a 1938, clasificados en dos grupos de productos, primarios y manufacturas. Baldwin presenta este estudio como un trabajo pionero y afirma que se comprende mejor que el elaborado por Tyszynski, a pesar de ser menos completo y detallado ${ }^{6}$.

Haberler (1958) comparó los resultados de todos estos trabajos y apreció cierta convergencia en las conclusiones fundamentales, aunque también percibió discrepancias significativas en algunos países y sobre todo en determinados artículos, debido en ciertos casos

${ }^{5}$ Lichtenberg evaluó el artículo de Baldwin y realizó algunas observaciones y sugerencias acerca del tema de los pesos que se han incorporado en el texto. Propuso la siguiente expresión:

$$
\begin{aligned}
\frac{\sum_{i} s_{i}^{1} \cdot X_{i}^{1}}{X^{1}}-\frac{\sum_{i} s_{i}^{0} \cdot X_{i}^{0}}{X^{0}}= & \frac{\sum_{i} s_{i}^{0} \cdot X_{i}^{1}}{X^{1}}-\frac{\sum_{i} s_{i}^{0} \cdot X_{i}^{0}}{X^{0}}+\frac{\sum_{i} s_{i}^{1} \cdot X_{i}^{0}}{X^{1}}-\frac{\sum_{i} s_{i}^{0} \cdot X_{i}^{0}}{X^{1}}+\frac{\sum_{i} s_{i}^{1} \cdot X_{i}^{1}}{X^{1}}- \\
& -\frac{\sum_{i} s_{i}^{0} \cdot X_{i}^{1}}{X^{1}}-\frac{\sum_{i} s_{i}^{1} \cdot X_{i}^{0}}{X^{1}}+\frac{\sum_{i} s_{i}^{0} \cdot X_{i}^{0}}{X^{1}}
\end{aligned}
$$

Los cuatro últimos sumandos son el componente interacción.Citado en Baldwin (1968), nota.17.

${ }^{6}$ Otras aportaciones significativas tras la Segunda Guerra Mundial recogidas en el artículo de Baldwin fueron las realizadas por Kindleberger (1956), Neisser y Modigliani (1953), Robinson (1953, 1954a, 1954b), Lewis (1952), Cairncross (1955), Cairncross y Faaland (1952). 
a las diferentes clasificaciones de productos utilizadas.Además fue crítico con la aplicación tan simplificada del método, pues argumentó que la distinción de dos factores exclusivos, estructura y competitividad, para descomponer exhaustivamente todos los cambios que sufre la cuota exportadora de un país es insuficiente.

Spiegelglas (1960) llevó a cabo un estudio para contrastar los resultados entre los años 1937-1956 adoptando la clasificación de mercancías de Tyszynski, y así poder comparar las conclusiones de ambos trabajos. Los grupos fueron dieciseis más un apartado de no especificados, y los países nueve, los once del análisis de Tyszynski excepto la India y Suiza. Añadió a las diferencias de cuotas halladas bajo el método, un concepto menos tratado, el de coeficiente de estabilidad, que medía el grado de estabilidad de las relaciones estructurales a lo largo del tiempo.

También Narvekar (1960) publicó el mismo año que Spiegelglas un estudio mucho más concreto en el que analizaba el espectacular incremento de las exportaciones japonesas en los años 1954-1958. Los grupos de productos en que clasificó las ventas de Japón fueron tres: los grupos 5,7 y $6+8$ de la clasificación $\mathrm{CUCl}$, es decir, productos químicos, maquinaria y equipo de transporte, y resto de manufacturas, respectivamente; y las áreas de referencia quince. Los efectos en los que descompuso el crecimiento fueron cuatro, ya que aplicó la versión ampliada del método, el efecto de crecimiento del comercio mundial, el efecto de expansión por el patrón de comercio según productos, el efecto de expansión por el patrón de comercio según mercados, y el efecto de incremento de la competitividad.

Romanis (1961) analizó para el mismo período que el trabajo anterior las causas que además de la competitividad vía precios explican las variaciones en la cuota exportadora de manufacturas norteamericana.El ejercicio lo realizó para los once países industriales líderes, y en un primer momento calculó los efectos estructura y competitividad, para ampliar el modelo posteriormente y obtener de esta manera más información sobre las causas del efecto demanda.

Lamfalussy (1963) para la Universidad de Yale, y Wells (1964) para la de Cambridge, realizaron dos trabajos donde se analizaba la estructura de la exportación británica. Ambos llevaron a cabo estudios comparativos, en concreto, el primero de ellos contrastó la evolución de las ventas de Reino Unido con las de Bélgica, Luxemburgo, 
Holanda, Francia, Italia y Alemania, los seis países fundadores de la CEE. Lamfalussy trató además los problemas de agregación de los mercados y la variabilidad asociada al orden en el cálculo de los efectos de estructura expuestos en el segundo punto.

Junz y Rhomberg (1965) elaboraron un estudio para los mismos once países de las aplicaciones anteriores, los más industrializados, en el período 1953-1963. Basándose también en la clasificación $\mathrm{CUCl}$ recogieron como Narvekar las secciones número 5,7 y $6+8$. Tras calcular los cuatro efectos concluyeron que aunque existe una clara asociación entre el movimiento de los precios relativos y la variación del volumen de las exportaciones de un país, resulta evidente que los efectos «no precio» juegan un papel importante en la misma. Profundizaron en la importancia de transformar los datos en moneda corriente a términos constantes para obtener una medida de las fuerzas demanda-oferta más ajustada a la realidad.

Stern (1967) dedicó su trabajo a analizar la gran expansión de las exportaciones italianas tras la Segunda Guerra Mundial. Los períodos seleccionados fueron cuatro: 1955-1959, 1959$1962,1962-1963,1963-1964$; los grupos de productos seis y las áreas a las que se dirigían estos bienes eran Norteamérica, Latinoamérica, CEE, EFTA, Resto de Europa Occidental, Europa del Este, Australia, Nueva Zelanda, Japón, Resto de Asia, Africa y Resto del mundo.

El modelo en el que se basó para el cálculo se formaliza a través de una expresión distinta a la de las cuotas, éste utiliza una nueva variable, $r$, definida como el cociente entre las exportaciones mundiales del año 1 y las del año 0 , y no como la tasa de crecimiento de dichas exportaciones, concepto adoptado posteriormente por el autor y Leamer en un trabajo conjunto. Además en el volumen de exportaciones mundiales excluyó las italianas $^{7}$. La identidad resultante es la siguiente ${ }^{8}$ :

7 Stern (1967) afirmó que las exportaciones del país analizado debían ser excluidas del cálculo de las exportaciones totales. En la aplicación él eliminó las exportaciones italianas. Otros trabajos posteriores que referencian este artículo o el elaborado por el autor y Leamer, proceden de igual manera y no tienen en cuenta las exportaciones nacionales. Se verá en los antecedentes españoles.

8 Se mantiene la notación. Excepto la definición de $r$.

$$
r=\frac{x^{1}}{x^{0}}
$$




$$
\begin{gathered}
\Delta x=r \cdot x^{0}-x^{0}+\left[\sum_{i} r_{i} x_{i}^{0}-r \cdot x^{0}\right]+ \\
+\left[\sum_{i} \sum_{j} r_{i j} \cdot x_{i j}^{0}-\sum_{i} r_{i} \cdot x_{i}^{0}\right]+\left[x^{1}-\sum_{i} \sum_{j} r_{i j} \cdot x_{i j}^{0}\right]
\end{gathered}
$$

Tres años más tarde, Stern y Leamer (1970) publicaron una discusión sobre el método y presentaron sus principales implicaciones teóricas y limitaciones. Es precisamente éste el estudio referido unas líneas antes. A lo largo de este año y en el siguiente vieron también la luz varios artículos elaborados por Richardson (1970, 1971a, 1971b, 1971c) acerca del método, todos fruto de su tesis doctoral sobre el Constant Market Shares Analysis. Los trabajos de estos tres autores son los más citados en las bibliografías de los estudios sobre el análisis de cuotas de mercado constantes, una referencia básica ${ }^{9}$.

Antes de finalizar la década de los setenta, Balassa $(1979)^{10}$ aplicó el método para calcular las elasticidades-renta de la demanda de exportaciones en un estudio sobre la composición y transformación de la estructura exportadora de los países industrializados en las décadas de los cincuenta y sesenta, como se ha visto escenario espacial y temporal común para la mayoría de los trabajos citados. Introdujo el concepto de exportaciones hipotéticas, siguiendo la idea de Tyszynski al hablar de cuotas hipotéticas, y las definió como las exportaciones que cada país realizaría al resto del mundo bajo el supuesto siguiente: que la competitividad inicial en cada grupo de productos permanezca constante.

Otro concepto expuesto por Balassa es el de elasticidad «aparente», que calculó como el cociente entre el crecimiento de las exportaciones hipotéticas y el crecimiento de la renta mundial, es decir, representa lo que incrementarían las exportaciones de un país bajo el supuesto de constancia de la compe-

9 Veánse estos estudios para un análisis detallado del método: elaboración teórica, distintos modelos, efectos derivados de cada uno de ellos, implicaciones y principales limitaciones de la técnica.

10 Balassa ya en 1962 había participado en un congreso en Estados Unidos sobre los factores que afectaban a la Balanza de Pagos norteamericana, y presentó un análisis de las transformaciones de la industria americana y su posible evolución. 
titividad en cada grupo, al producirse un incremento de una unidad monetaria en la renta mundial. Recoge por lo tanto el efecto demanda mundial.

Ya en la década de los ochenta, Feeny (1982) llevó a cabo un estudio sobre las infraestructuras y el desarrollo comercial de Tailandia y utilizó la metodología de participación constante aplicada a unas producciones muy concretas. Durante el período 1850-1940 el país respondió dinámicamente al proceso de apertura exterior y crecimiento de la demanda mundial de las exportaciones de arroz, madera de teca, caucho y estaño. El trabajo dio luz acerca de los importantes cambios ocurridos en la economía tailandesa en los primeros cuarenta años del siglo actual debido al espectacular comportamiento de la cuota exportadora de arroz. Los tres efectos derivados del análisis resultaron positivos, pero el efecto demanda dominó sobre los efectos competitivo e interacción.

Fagerberg y Sollie (1987) desarrollaron una versión del método que añadía dos nuevos efectos a los ya definidos: la capacidad de cada país para adaptar su estructura exportadora tanto a cambios en la composición de productos como de mercados de las importaciones mundiales. Realizaron el ejercicio para una muestra de veinte países de la OCDE para el período 1961-1983 y obtuvieron resultados interesantes. Los cambios estructurales contribuyeron positivamente al crecimiento de las exportaciones de los países más avanzados, y, negativamente, para los menos desarrollados. Sin embargo, los primeros se adaptaron peor a las nuevas circunstancias del mercado y, en general, los segundos ganaron cuota de mercado a costa de ellos.

Dos años más tarde Hoen y Wagener (1989) analizaron las repercusiones de la reforma llevada a cabo en Hungría en la evolución de las exportaciones del país a los mercados de la OCDE. El comportamiento comercial húngaro es comparado con el de otros países del este europeo, los cuales sufrieron pérdidas de cuota de mercado más elevadas durante los setenta y ochenta. Este desarrollo tan débil se debió a un efecto competitivo negativo y a la baja capacidad de adaptación a las condiciones del mercado mundial.

En los años noventa aparecen tres nuevos artículos sobre el análisis de cuotas de mercado constantes aplicado a tres paí- 
ses concretos: Alemania, Reino Unido y Japón, aunque en el último caso el estudio se refiere a las cuotas de importación y de un solo producto, el trigo. El autor del mismo es AhmadiEsfahani (1995). En el primero de ellos Oldersma y van Bergeijk (1993) demuestran que la moneda utilizada para expresar las exportaciones de un país influye en los resultados derivados del método, e ilustran su teoría con un estudio empírico de las ventas exteriores alemanas entre 1989 y 1991. El segundo, elaborado por Irwin (1995), explica el declinar de las exportaciones británicas a partir de 1870, atribuido hasta entonces a la incapacidad para trasladar recursos desde sectores de bajo crecimiento a otros de mayor desarrollo y mejores perspectivas. Sin embargo, el autor a través del análisis CMS observa pérdidas de cuota muy significativas en los productos y mercados tradicionales, y las responsabiliza de la debilidad comercial británica en la época.

\section{Antecedentes españoles}

Fue un destacado miembro entonces de la Universidad de Kiel el primer autor que planteó la aplicación de este método al caso español. Donges $(1971,1972,1976)$ utilizó este instrumento para analizar el crecimiento de nuestro comercio exterior en los años sesenta dentro de un marco más amplio: una interpretación general de la industrialización española. Estudió la evolución de las exportaciones industriales desde 1962 hasta 1972, una década en la que la economía española abrió sus puertas al exterior y acabó con el sistema autárquico vivido en los años cuarenta y cincuenta.

El modelo elegido para realizar los cálculos fue el mismo que presentó Stern en su trabajo, definió igual $r$ y excluyó también del cómputo de exportaciones mundiales a las españolas.

Los grupos de productos manufacturados que se incluyeron fueron, como en otros trabajos extranjeros que se han mencionado, los grupos 5 (productos químicos), 7 (maquinaria y equipo de transporte), y 6+8 (otras manufacturas), de la clasificación CUCl. El grado de agregación es alto, en consonancia con los datos disponibles, ya que la Organización de las Naciones 
Unidas no cuenta con series de comercio internacional para esos años a un nivel más detallado por países y productos ${ }^{11}$.

Las áreas importadoras fueron quince: Estados Unidos, Canadá, Latinoamérica, CEE (seis), AELE, Europa Oriental, Europa meridional (excluida España), Sudáfrica, otros países africanos, Cercano Oriente, economías planificadas de Asia central, Japón, otros países asiáticos, Australia, Nueva Zelanda y el Resto del mundo.

Donges llegó a la conclusión de que la industria española había mejorado durante ese período su competitividad externa. Si hubiera mantenido constante su cuota de mercado en el comercio mundial las exportaciones de manufacturas se habrían incrementado tan sólo en 921 millones de dólares y no en los 2300 millones que crecieron. En la descomposición por efectos el de demanda supone un $23,5 \%$, el de productos un $-1,4 \%$, el de mercados un $18,5 \%$ y el competitivo un $59,4 \%$. Todo esto calculado para precios corrientes.

Para ámbitos supranacionales se encuentran dos trabajos realizados también en 1982, uno de la Comisión Europea y otro de las Naciones Unidas. El primero para la etapa 1970-1980, y el segundo para los períodos 1970/72-1976/78. En ambos se analizó el comercio exterior de España, aunque resulta difícil comparar entre ellos y otros ensayos del caso español debido a los diferentes años y ámbitos de países considerados en cada estudio.

A pesar de estas dificultades sí se observa cierta convergencia en las conclusiones más importantes: en los años setenta el efecto competitividad representa un porcentaje elevado en la explicación del crecimiento de las exportaciones españolas, y superior al que en esos años han experimentado otros países europeos o del entorno económico de España; además la contribución debida al efecto demanda ha sido también decisiva, comportamiento normal tratándose España de un país pequeño y medianamente abierto al exterior en esos años; la elección de mercados destino de las ventas contribuye positivamente, sin embargo el efecto composición de la cesta de productos expor-

${ }^{11}$ Este es un problema general de los autores al aplicar el método a un caso particular. Las estadísticas de Naciones Unidas presentan un alto grado de desagregación cuando se trata de productos solamente o de países. Sin embargo, la clasificación cruzada (productos y países) aparece muy agregada. 
tados es generalmente muy pequeño, incluso a veces negativo, confirmando la especialización española en sectores de demanda débil y poco dinamismo.

Unos años más tarde Palafox (1986) recurrió a diferencia de Donges al modelo simplificado ${ }^{12}$ y obtuvo además de los dos efectos básicos, demanda y competitividad, un efecto interacción. Analizó el crecimiento de las ventas exteriores españolas en el período 1913-1928, para ello llevó a cabo su ejercicio a precios corrientes y utilizó las cifras oficiales del comercio exterior español. Los resultados obtenidos a partir de estas series oficiales y del valor del comercio europeo calculado por Svennilson, afirman que España incrementó su cuota de mercado tanto con relación a Europa como al mundo. En el marco europeo el efecto competitivo supuso un $23,9 \%$ y el efecto interacción contribuyó en un 14,6\%. Palafox centró su argumentación en la competitividad de los productos alimenticios, especialmente cítricos y vino, que representaban en esos años casi la cuarta parte del total de las exportaciones del país.

Sería Prados (1988) quien realizase un nuevo ejercicio para el mismo período de tiempo, pero con nuevos datos más fiables sobre el comercio exterior español y europeo. Expuso que si Palafox hubiera usado estas nuevas estimaciones españolas y los cálculos para Europa de Bairoch, los efectos competitivo e interacción sumados hubieran supuesto un porcentaje inferior al anterior, un $25,7 \%$ y no un $38,5 \%$. Luego la ganancia de cuota hubiera sido bastante menor. Además repitió los cálculos realizándolos esta vez en términos reales. De esta forma los resultados fueron muy distintos: la evolución de las exportaciones dependía ahora casi únicamente del efecto demanda.Esto era más coherente con la caída del volumen exportado que había sufrido la balanza comercial de nuestro país en el período tratado por Palafox ${ }^{13}$. Prados también analizó el crecimiento de las exportaciones de cítricos y vino individualmente debido a la importancia de estos productos en el total, y los resultados en este caso sí atribuyeron un papel muy importante a la variable competitividad.

12 Estos modelos más sencillos suelen emplearse cuando los datos disponibles no permiten un estudio exhaustivo más desagregado.

${ }^{13}$ El propio autor señaló que esta contradicción había sido denunciada por Maluquer de Motes en 1987. 
Iniciada ya la nueva década Alonso (1990), en un estudio sobre el sector exterior español y los factores de cambio, descompuso la evolución de las exportaciones en los cuatro componentes estadísticos complementarios para el período que transcurre desde 1970 hasta el año previo a la incorporación de España en las Comunidades Europeas, 1985. Durante esos quince años las ventas españolas al exterior crecieron en términos reales a una tasa media anual del 8,2\%, que duplica la correspondiente al comercio mundial. Las consecuencias de este comportamiento, pese a las dificultades internacionales del período, dos crisis energéticas, fueron muy positivas: en primer lugar, se incrementó la cuota de mercado española en el total mundial; en segundo lugar, continuó el proceso de apertura emprendido por la economía española tras el Plan de Estabilización; y por último, se redujo la brecha de nuestro comercio exterior. Utilizó, como Stern y luego Donges, la versión más amplia del modelo en la presentación alternativa a la del empleo de las cuotas, con datos de la ONU y la OCDE. Los resultados ponen en evidencia que los factores de oferta explican más del $60 \%$ del crecimiento de las exportaciones industriales en esos tres lustros, porcentaje que todavía es mayor si los cálculos se hacen en términos reales; y que los factores de demanda también han tenido un papel importante.

Al año siguiente Tena (1991) analizó los cambios reales de las exportaciones españolas e italianas también mediante la técnica de cuotas de mercado constantes, pero en este caso retrocedió como Palafox y Prados a etapas anteriores: 1890-1912, 1913-1928 y 1948-1958, y calculó la participación tanto para el total europeo como para el mundial. Realizó el ejercicio a precios constantes y utilizó una de las formas simplificadas, sin profundizar en el análisis de los grupos de productos o mercados que han contribuido a la expansión de las ventas al exterior debido a una especialización favorable. Para España los resultados arrojados en todos los períodos desvelan unas aportaciones negativas muy importantes tanto del efecto competitivo como del efecto interacción. Sólo el efecto demanda es además de significativo un factor positivo a la hora de explicar el crecimiento de las exportaciones de nuestro país en esos años anteriores a la década de los sesenta. 
Finalmente, Asensio (1995) en su tesis doctoral aplicó el análisis de las cuotas de mercado a las exportaciones españolas del período 1951-1958, último estudiado por Tena. El trabajo tomó como área de referencia el conjunto de países de la OECE, excepto Grecia y Suiza, y calculó los efectos mercado, competitivo e interacción. Tanto a precios corrientes como constantes, los resultados obtenidos concluyeron que durante los años cincuenta el incremento de las exportaciones se debió fundamentalmente al aumento de la demanda mundial, mientras que el efecto competitivo frenó el nivel de ventas exteriores. De hecho, España perdió cuota de mercado respecto al grueso de los países desarrollados, es decir, redujo su competitividad estructural con relación a estos países durante los ocho años analizados.

\section{Referencias bibliográficas}

ADAMS, F. G., H.EGUCHI y MEYER-ZU-SCHLOCHTERN, F. M. (1969): «An econometric analysis of international trade: an interrelated explanation for imports and exports of OCDE countries». Organitation for Economic Cooperation and Development, París.

AHMADI-ESFAHANI, F. (1995): «Wheat Market Shares in the Presence of Japonese Import Quotas», Journal of Policy Modeling, 17, June 1995, pp. 315-323.

ALONSO, J. A. (1990): «Comercio exterior: factores de cambio» en GARCÍA DELGADO, J. L., (eds.), La Economía española de la transición y la democracia, CIS.

ASENSIO CASTILLO, M. J. (1995): El proceso de apertura exte rior de los cincuenta y el arancel de 1960, tesis doctoral dirigida por el Dr. D. José M ${ }^{a}$ Serrano Sanz y leída en la Universidad de Zaragoza.

ASHBY, L. D. (1968): The shift and share analysis: a reply, Southern Economic Journal, 34, pp.423-425.

BALASSA, B. (1979): «Export Composition and Export Performance in the Industrial Countries, 1953-1971», Applied Economics, pp. 604-607.

BALDWIN, R.E. (1958): «The Commodity Composition of Trade: Selected Industrial Countries, 1900-1954», Review 
of Economics and Statistics, XL (February 1958, Supplement), pp. 50-71.

BOWEN, H. P. y PELZMAN, J. (1984): «US export competitiviness: 1962-1977», Applied Economics, 16, pp. 461-473.

BROWN, H. J. (1969): Shift and share projections of regional economic growth: an empirical test, Journal of regional Science 9, pp.1-17.

CAIRNCROSS, A. K. (1955): "World Trade in Manufactures Since 1900», Economia Internazionale, Vol.VIII, no 4, 1955.

CAIRNCROSS, A. K. y FAALAND, J. (1952): «Long-Tern Trends in Europe's Trade», Economic Journal, March 1952.

COMISIÓN ECONÓMICA PARA EUROPA (1982): «The relative performance of south european exports of manufactures to OCDE countries in 1970s. An analysis of demand factors and competitiviness", Economic Bulletin for Europe, vol. 34.

DONGES, J. B. (1971): «From an Autarchic Towards a Cauteously Outward-Looking Industrialization Policy: The Case of Spain», Weltwirtschaftliches Archiv, 107, pp. 33-72.

- (1972): «Spain's industrial exports - An anlysis of demand and supply factors", Weltwirtschaftliches Archiv, 108, pp.191232.

- (1973): «Shaping Spain's Export Industry», World Development, vol. 1, pp. 19-37.

-(1976): La industrialización en España, Oikos, Barcelona

FAGERBER, J. y SOLLIE, G. (1987), «The method of constant market shares analysis reconsidered», Applied Economics, 19, pp. 1571-1583.

FEENY, D. (1982): «Infraestructure Linkages and Trade Performance: Thailand 1900-1940», Explorations in Economic History, XIX, 1, pp. 1-27.

FEIRRARA, M. P.y RAIMENT, P. (1984): «Exports of manufactures from South European countries", Journal of World Trade Law, 18, pp 235-251.

FLEMING, J. M. y TSIANG, S. C. (1958): «Changes in Competitive Strenght and Export Shares of Major Industrial Countries", International Monetary Fund, Staff Papers, V (August 1958), pp. 218-248.

HABERLER, G. (1958): «Introduction» to the Papers and Abstracts of Papers at a Universities-National Bureau Committee for Economic Research Conference on Problems 
in International Economics, Review of Economics and Statistics, XL (February 1958, Supplement), pp. 3-9.

HOEN, H. y WAGENER, H. (1989): «Hungary's Exports to the OCDE: A Constant Market Shares Analysis», Acta Oeconomica, 40 (1-2), pp. 65-77.

HOUSTON, D.B.(1967): «The shift and share analysis of regional growth: a critique, Southern Economic Journal, 33, pp.577-581.

IRWIN, D. (1995): «The Lion's Share: Britain's Export Performance Revisited, 1899-1929», Structural Change and Economic Dynamics, 6, March 1995, pp. 97-109.

JEPMA, C. (1989): «Extensions of the Constant Market Shares Analysis with an Application to Long Term Export Data of Developing Countries», en WILLIAMSON, J. y PANCHAMU$\mathrm{KHI}, \mathrm{V}$. (eds), The balance between industry and agriculture in economic development: Proceedings of the Eighth World Congress of the International Economic Association, Delhi, India.Vol.2. Sector proportions. New York, 1989, pp. 129-143. JUNZ, H. B. y RHOMBERG, R. R. (1965): «Prices and Export Performance of Industrial Countries, 1953-1963", International Monetary Fund, Staff Papers, XII (July 1965), pp. 224-269.

KINDLEBERGER, C. P. (1956): The Terms of trade, A European Case Study, Wiley.

KREININ, M. E. (1967): "Price Elasticities in International Trade», Review of Economics and Statistics, 44 (November 1967), pp. 510-516.

LAMFALUSSY, A. (1963): The United Kingdom and the Six. Homewood: Richard D. Irwin, Inc., for Yale University Growth Center, esp. pp. 47-58 and pp. 137-140.

LEAMER, E. E., y STERN, R. M. (1970): Quantitative International Economics (Boston: Allyn and Bacon).

LEWIS, A. (1952): «World Production, prices and Trade, 18701960», Manchester School, May 1952.

LICHTENBERG, R. M. (1960): One-tenth of a nation, Harvard University Press, Cambridge, Mass.

MAGEE, S. P. (1975): «Prices, Income, and Foreign Trade», en Peter B. Kenen (ed.), International Trade and Finance: Frontiers for Research (Cambridge University Press). 
NARVEKAR, P.R. (1960): «The Role of Competitiveness in Japan's Export Performance, 1954-1958», International Monetary Fund, Staff Papers, VIII (November 1960), pp. 85100.

NEISER H. y MODIGLIANI, F. (1953): National Incomes and International Trade, University of Illinois Press.

OLDERSMA, H. yVAN BERGEIJK, P. (1993): «Not So Con\$tant! The Constant Market Shares Analysis and the Exchange Rate», De Economist, 141 (3), pp. 380-401.

ONUDI (1982): «Hanbook of industrial statistiques», NuevaYork. PALAFOX, J. (1986): “Comercio Exterior y Vía Nacionalista. Algunas consideraciones», en García Delgado J.L.(ed.): La crisis de la Restauración. España, entre la Primera Guerra Mundial y la Segunda República, Madrid s. XXI, pp. 186-192. PRADOS DE LA ESCOSURA, L. (1988): De imperio a nación. Crecimiento y atraso económico en España (1780-1930), Madrid, Alianza editorial.

RICHARDSON, D. J. (1970): Some Problems in the «ConstantMarket-Shares Analysis of Export Growth», Research Seminar in International Economics Discussion Paper nํ19, University of Michigan.

- (1971a): "Altering the World of Competitors in a ConstantMarket-Shares Analysis of Export Growth», Research Seminar in International Economics Discussion Paper no 36, University of Michigan.

- (1971b): «Constant-Market-Shares Analysis of Export Growth», Journal of International Economics 1 (May 1971), pp. 227-239.

- (1971c): «Some sensitivity Tests for a Constant-MarketShares Analysis of Export Growth», Review of Economics and Statistics, 53 (August 1971), pp. 300-304.

ROBINSON, E. A. G. (1953): «The future of British Imports», Three Banks Review, March 1953.

— (1954a): «The problem of Living Within Our Foreign Earnings», Three Banks Review, March 1954.

- (1954b): «The Changing Structure of the British Economy», Economic Journal, September 1954.

ROMANIS, A. (1961): "Relative Growth of Exports of Manufactures of Unites States and Other Industrial 
Countries», International Monetary Fund, Staff Papers, VIII (May 1961), pp. 241-273.

SARNO, D. (1988): "Constant Market Shares Analysis: Un modelo alternativo", Studi Economici, 43 (34), pp. 25-52.

SPIEGELGLAS, S. (1959): «World Exports of Manufactures, 1956 vs. 1937»", The Manchester School, XXVII (May 1959), pp. $111-139$.

STERN, R. M. (1967), Foreign Trade and Economic Growth in Italy. New York: Frederick A. Praeger, Chap.2.

SVENNILSON, I. (1954): Growth and Stagnation in the European Economy. Geneva: United Nations.

TENA, A. (1991): «Protección y competitividad en España e Italia, 1890-1960", en Prados de la Escosura, L. y Zamagni, V. (eds.), El desarrollo económico en la Europa del Sur: España e Italia en perspectiva histórica, Madrid, Alianza.

TYSZYNSKI, H. (1951): «World Trade in Manufactured Commodities, 1899-1950", The Manchester School, XIX (September 1951), pp. 272-304.

UTNE, A. (1984): «The EFTA countries'export performance for manufactured goods 1970-1982", Occasional Paper № 7, EFTA, Geneva. 